\title{
Orbital motion in T Tauri binary systems ${ }^{\star, \star \star}$
}

\author{
J. Woitas ${ }^{1,2}$, R. Köhler ${ }^{3,2}$, and Ch. Leinert ${ }^{2}$ \\ 1 Thüringer Landessternwarte Tautenburg, Sternwarte 5, 07778 Tautenburg, Germany \\ 2 Max-Planck-Institut für Astronomie, Königstuhl 17, 69117 Heidelberg, Germany \\ 3 Center for Astrophysics and Space Sciences, University of California, San Diego, 9500 Gilman Drive, \\ La Jolla, CA 92093-0424, USA
}

Received 6 June 2000 / Accepted 9 January 2001

\begin{abstract}
Using speckle-interferometry we have carried out repeated measurements of relative positions for the components of $34 \mathrm{~T}$ Tauri binary systems. The projected separation of these components is low enough that orbital motion is expected to be observable within a few years. In most cases orbital motion has indeed been detected. The observational data is discussed in a manner similar to Ghez et al. (1995). However, we extend their study to a larger number of objects and a much longer timespan. The database presented in this paper is valuable for future visual orbit determinations. It will yield empirical masses for $\mathrm{T}$ Tauri stars that now are only poorly known. The available data is however not sufficient to do this at the present time. Instead, we use short series of orbital data and statistical distributions of orbital parameters to derive an average system mass that is independent of theoretical assumptions about the physics of PMS stars. For our sample this mass is $2.0 M_{\odot}$ and thus in the order of magnitude one expects for the mass sum of two T Tauri stars. It is also comparable to mass estimates obtained for the same systems using theoretical PMS evolutionary models.
\end{abstract}

Key words. stars: binaries: visual - stars: pre-main sequence - techniques: interferometric

\section{Introduction}

The mass is the most fundamental parameter of a star, because, to a large extent, it determines its structure and evolution. Therefore, it is a major problem for our understanding of pre-main sequence (PMS) evolution that at this time there are no reliable empirical mass determinations for young stars with $M<1 M_{\odot}$. Mass estimates for $\mathrm{T}$ Tauri stars are usually based on comparisons of their position in the Hertzsprung-Russell diagram (HRD) with theoretical PMS evolutionary models, which means that they are affected by the (unknown) uncertainties within these models. Moreover, it is not possible to rate the quality of different sets of PMS models by comparison to observational data.

Send offprint requests to: J. Woitas, e-mail: woitas@tls-tautenburg.de

* Based on observations collected at the German-Spanish Astronomical Center on Calar Alto, Spain, and at the European Southern Observatory, La Silla, Chile.

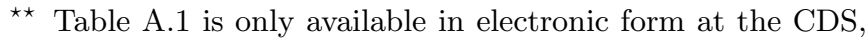
via anonymous ftp to cdsarc.u-strasbg.fr (130.79.128.5), or via

http://cdsweb.u-strasbg.fr/cgi-bin/qcat?J/A+A/369/249
For this reason, empirical mass determinations for young stars are highly desirable. Binary stars offer a unique possibility to do this, because the system mass is known as soon as the orbital parameters are determined. There are many binaries among $\mathrm{T}$ Tauri stars in nearby star-forming regions (SFRs). Most of them have been detected during the last decade by high-angular resolution surveys in the near infrared (NIR) (for an overview of this topic, see the review by Mathieu et al. (2000) and references therein).

The first reliable empirical masses of PMS stars were given by Casey et al. (1998) for the components of the eclipsing double-lined spectroscopic binary (ESB2) TY CrA. These masses are $M_{1}=3.16 \pm 0.02 M_{\odot}$ and $M_{2}=1.64 \pm 0.01 M_{\odot}$. The secondary mass is consistent with the predictions of PMS models from D'Antona \& Mazzitelli (1994) and also Swenson et al. (1994). The primary is already close to the main sequence. The lowestmass PMS stars with empirically determined masses thus far known are the components of RX J0529.4+0041. For this ESB2, Covino et al. (2000) determined the masses $M_{1}=1.25 \pm 0.05 M_{\odot}$ and $M_{2}=0.91 \pm 0.05 M_{\odot}$. They concluded that these masses are in good agreement with with the Baraffe et al. (1998) and Swenson et al. (1994) 
models, but less consistent with sets of PMS tracks from D'Antona \& Mazzitelli (1994) and Palla \& Stahler (1999). Because of the relatively high masses, these results cannot be used to check the PMS models for K- or M-dwarfs and objects with masses below the hydrogen burning mass limit at $\approx 0.075 M_{\odot}$.

In this paper we will follow the approach of Ghez et al. (1995, herafter G95). Using NIR speckle interferometry, they obtained repeated measurements for the relative astrometry of the components in $20 \mathrm{~T}$ Tauri binary systems. In this way they showed that in most of these systems, orbital motion can be determined. From short pieces of orbital data and the statistical distribution of orbital parameters, they have derived the average system mass of $1.7 M_{\odot}$, which is in the order of magnitude expected for the mass of two $\mathrm{T}$ Tauri stars.

We present similar data for $34 \mathrm{~T}$ Tauri binary systems and in this way increase the object list and also the observational time base. An overview of our observations and data reduction is given in Sect. 2. The results are presented in Sect. 3, discussed in Sect. 4 and summarized in Sect. 5 .

\section{Observations and data reduction}

\subsection{The sample}

We have repeatedly observed 21 systems in Taurus-Auriga detected as binaries by Leinert et al. (1993) and Köhler \& Leinert (1998) and 11 systems in Scorpius-Centaurus detected by Köhler et al. (2000). Furthermore, we reobserved two binaries found by Ghez et al. (1997a). These are HM Anon in the Chamaeleon I association, and HN Lup in the Lupus SFR. We combine our data with results taken from literature (see Sect. 2.6). In particular twelve systems discussed by G95 are also objects of our study.

\subsection{Observations}

The objects in Taurus-Auriga were observed with the 3.5 m-telescope on Calar Alto. After September 1993 these observations used the NIR array camera MAGIC. Previous measurements were obtained with a device for one-dimensional speckle interferometry described by Leinert \& Haas (1989). The observations of young binaries in southern SFRs were carried out at the ESO New Technology Telescope (NTT) at La Silla that is also a $3.5 \mathrm{~m}$-telescope. The instrument used for these observations was the NIR array camera SHARP I of the Max-Planck Institute for Extraterrestrial Physics (Hofmann et al. 1992). Both cameras are capable of obtaining fast sequences of short exposures with integration times $\tau \approx 0.1 \mathrm{~s}$, which is crucial for the applied data reduction process (see Sect. 2.3).

Most of the data were obtained in the $K$-band at $\lambda=2.2 \mu \mathrm{m}$. Some observations used the $J$-band at $\lambda=1.25 \mu \mathrm{m}$ and the $H$-band at $\lambda=1.65 \mu \mathrm{m}$. In these cases the main goal of the observations was to obtain resolved photometry of the components at those wavelenghts. In the course of data reduction we could however show that highly precise relative astrometry can also be derived from observations in $J$ and $H$ (see Sect. 2.4 for the determination of binary parameters).

\subsection{Speckle interferometry}

At the presumed distance of the objects (between 140 and 190 pc, see Sect. 3.1 and Table 1) orbital motion measurable within a few years can only be expected for the closest pairs with projected separations of $d \leq 0^{\prime \prime} .5$. Therefore, high angular resolution techniques are necessary that overcome the effects of atmospheric turbulence and yield the diffraction-limited information about the objects. For this purpose, we have used speckle interferometry.

Sequences of $\approx 1000$ short exposures $(\tau \approx 0.1 \mathrm{~s})$ are taken for the object and a nearby point source, the reference star. The integration time is shorter than the coherence time of the turbulent atmosphere, so the turbulence is "frozen", and the images are noisy, but principally diffraction limited. After Fourier transforming these "data cubes", the power spectrum of the image is deconvolved with that of the reference star to obtain the modulus of the complex visibility. The phase is reconstructed using the Knox-Thompson algorithm (Knox \& Thompson 1974) and the bispectrum method (Lohmann et al. 1983). The complex visibility is the Fourier transform of the object brightness distribution. For a sufficiently bright object it will contain the diffraction-limited information.

\subsection{Determination of binary parameters}

Modulus and phase of the complex visibility are characteristic strip patterns for a binary, as shown in Fig. 1 (first row) for the XZ Tau system. The position angle of the companion is orientated perpendicular to these patterns and towards higher values of phase. The distance between two strips is inversely proportional to the projected separation of the components, and the amplitude of the patterns denotes the flux ratio. Position angle, projected separation and flux ratio are determined by constructing an artificial complex visibility from a set of these parameters (second row in Fig. 1) and fitting it to the data (third row in Fig. 1). This fit uses the Amoeba algorithm (Press et al. 1994). The errors of the binary parameters are estimated by applying this fitting procedure to different subsets of the data.

\subsection{Pixel scale and detector orientation}

To obtain position angle and projected separation as astronomical quantities they must be transformed from the array onto the sky by calibrating them with pixel scale and orientation of the detector. Minimizing the 

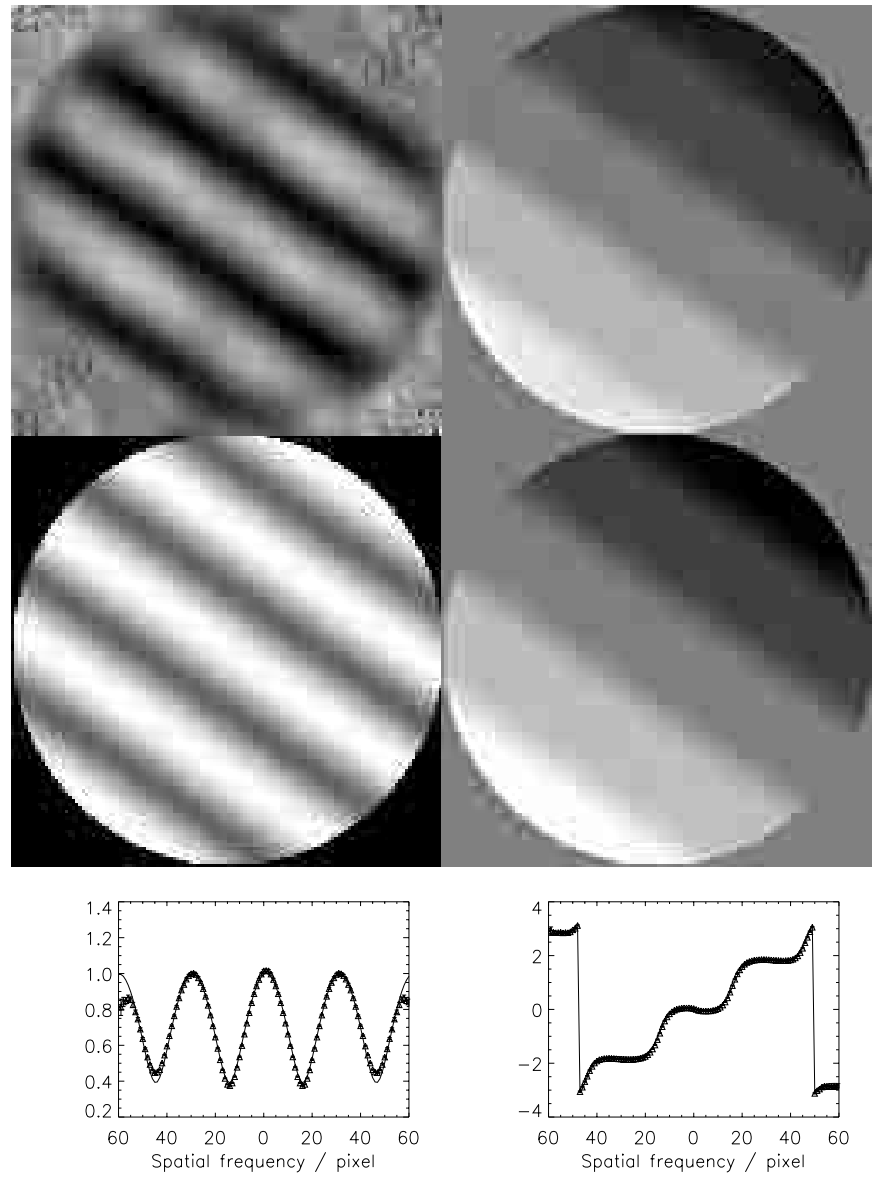

Fig. 1. The first row shows modulus (left) and bispectrumphase (right) of the complex visibility for the binary XZ Tau, derived from data obtained on 29 Sep. 1996 at the $3.5 \mathrm{~m}$ telescope on Calar Alto with the NIR array camera MAGIC at $\lambda=2.2 \mu \mathrm{m}$. The second row is the modulus and phase of an artificial complex visibility that is fitted to the data. This fit is indicated in the third row in a one-dimensional projection towards the connection line of the components (perpendicular to the strip patterns in Fourier space). The circle around the strip patterns corresponds to the Nyquist spatial frequency, $7.0 \operatorname{arcsec}^{-1}$ for the adopted pixel scale

uncertainties that result from this calibration is crucial for a highly accurate determination of the components' relative astrometry that is proposed here. We determine pixel scale and detector orientation from astrometric fits to images of the Orion Trapezium cluster where precise astrometry has been derived by McCaughrean \& Stauffer (1994). Typical errors are $0.1^{\circ}$ for the detector orientation and $10^{-4} \mathrm{arcsec} /$ pixel for the pixel scale that is $\approx 0^{\prime \prime} .07 /$ pixel for MAGIC and $\approx 0$ ". $05 /$ pixel for SHARP I.

This precise calibration exists for all observations obtained by the authors since July 1995. For calibrating data from previous observing runs we used visual binary stars with well-known orbits. We observed these stars afterwards and calibrated their separation and position angle with the help of the Trapezium cluster. Thus, we have placed all our (two-dimensional) speckle observations into a consistent system of pixel scale and detector orientation.
Table 1. Adopted distances to stars in nearby SFRs

\begin{tabular}{lll}
\hline SFR & Distance [pc] & Reference \\
\hline Taurus-Auriga & $142 \pm 14$ & Wichmann et al. (1998) \\
Scorpius-Centaurus & $145 \pm 2$ & de Zeeuw et al. (1999) \\
Chamaeleon I & $160 \pm 17$ & Wichmann et al. (1998) \\
Lupus & $190 \pm 27$ & Wichmann et al. (1998) \\
\hline
\end{tabular}

\subsection{The database}

In Table A.1 the derived position angles, projected separations and flux ratios are presented. For the subsequent analysis (Sect. 3) the relative positions are combined with data taken from literature. Twelve of our systems have also been discussed by G95. For some binaries there are additional measurements obtained by HST imaging, the HST Fine Guidance Sensors or adaptive optics (see references in Table A.1). The finding that our relative astrometry fits very well with that obtained by other groups further supports the idea that the position angles and projected separations are as precise as is indicated by the errors given in Table A.1.

\section{Results}

\subsection{Relative motion of the components}

In Fig. A.1 the relative positions of the components at different epochs are shown in cartesian and polar coordinates. These plots are only given for the 23 out of our 34 systems for which there are at least three data points. To obtain a simple approximation of the relative velocity we applied weighted linear fits to this data. For the 11 systems with only two observations we simply connect the two data points and derive the error of the relative velocity from the uncertainties of the two separations and position angles.

For a quantitative analysis, the relative velocities must be transformed to an absolute length scale. This requires knowing the distances of the discussed objects. We adopt distances to the SFRs that are the mean of all Hipparcos distances derived for members of the respective association. The values and references are given in Table 1 . There remains, however, an uncertainty, because distances of individual objects may be different from these mean values. To take this into account, we assume that the radial diameters of the SFRs are as large as their projected diameters on the sky. The latter quantity can be estimated to be $\approx 20^{\circ}$ for Taurus-Auriga (see Fig. 1 in Köhler \& Leinert 1998) as well as Scorpius-Centaurus (see Fig. 1 in Köhler et al. 2000). Concerning the mean distances from Table 1, this corresponds to a diameter of $50 \mathrm{pc}$. Thus we will assume $\pm 25 \mathrm{pc}$ as the uncertainty for the distance of an individual system, which is an upper limit: more than two thirds of the stars will be within $\pm 15 \mathrm{pc}$ for an even distribution. 
Table 2. Projected relative velocities of the companions with respect to the primaries in cartesian and polar coordinates. The adopted $v$ is the mean of the total velocities derived from $\left(v_{x}, v_{y}\right)$ and $\left(v_{\rho}, v_{\phi}\right)$. Note that $v_{\rho}$ is given with respect to the main component and not relative to the observer

\begin{tabular}{|c|c|c|c|c|c|c|}
\hline System & $v_{x}\left[\mathrm{~km} \mathrm{~s}^{-1}\right]$ & $v_{y}\left[\mathrm{~km} \mathrm{~s}^{-1}\right]$ & $v_{\rho}\left[\mathrm{km} \mathrm{s}^{-1}\right]$ & $v_{\phi}\left[\mathrm{km} \mathrm{s}^{-1}\right]$ & $v\left[\mathrm{~km} \mathrm{~s}^{-1}\right]$ & $\bar{d}[\mathrm{AU}]$ \\
\hline V 773 Tau & $-1.35 \pm 2.47$ & $15.64 \pm 6.87$ & $-8.37 \pm 6.82$ & $8.41 \pm 2.83$ & $13.78 \pm 7.34$ & 14.9 \\
\hline $\mathrm{LkCa} 3$ & $4.54 \pm 1.92$ & $1.16 \pm 1.07$ & $-0.60 \pm 1.49$ & $-2.98 \pm 1.62$ & $3.86 \pm 2.20$ & 68.7 \\
\hline FO Tau & $3.67 \pm 1.63$ & $-5.62 \pm 1.52$ & $-1.48 \pm 1.36$ & $5.63 \pm 1.72$ & $6.27 \pm 2.21$ & 22.3 \\
\hline CZ Tau & $-4.83 \pm 1.46$ & $-1.97 \pm 2.22$ & $-2.72 \pm 2.27$ & $4.98 \pm 1.43$ & $5.45 \pm 2.67$ & 45.3 \\
\hline FS Tau & $-9.26 \pm 2.87$ & $2.06 \pm 1.44$ & $-1.88 \pm 1.54$ & $6.61 \pm 2.77$ & $8.18 \pm 3.19$ & 35.6 \\
\hline FW Tau & $8.11 \pm 2.12$ & $-7.69 \pm 2.22$ & $-7.77 \pm 2.24$ & $6.87 \pm 1.67$ & $10.77 \pm 2.93$ & 15.8 \\
\hline $\mathrm{LkH} \alpha 331$ & $-3.77 \pm 2.21$ & $2.51 \pm 1.04$ & $-1.55 \pm 1.65$ & $1.76 \pm 1.77$ & $3.44 \pm 2.43$ & 40.3 \\
\hline XZ Tau & $2.14 \pm 1.03$ & $4.25 \pm 1.32$ & $-0.35 \pm 0.50$ & $-5.03 \pm 1.52$ & $4.90 \pm 1.64$ & 43.2 \\
\hline HK Tau G2 & $0.41 \pm 0.34$ & $-2.06 \pm 1.92$ & $5.00 \pm 1.80$ & $-2.05 \pm 1.92$ & $3.75 \pm 1.95$ & 26.3 \\
\hline GG Tau Aa & $-0.62 \pm 0.69$ & $-6.59 \pm 1.82$ & $0.41 \pm 0.72$ & $-4.71 \pm 1.80$ & $5.67 \pm 1.94$ & 35.8 \\
\hline UZ Tau/w & $3.20 \pm 1.31$ & $1.36 \pm 1.24$ & $1.21 \pm 1.32$ & $2.76 \pm 1.24$ & $3.24 \pm 1.81$ & 50.9 \\
\hline GH Tau & $8.61 \pm 2.96$ & $-2.97 \pm 2.14$ & $-1.42 \pm 3.18$ & $-3.19 \pm 1.92$ & $6.30 \pm 3.68$ & 45.0 \\
\hline Elias 12 & $-13.79 \pm 3.95$ & $-0.65 \pm 1.71$ & $-6.05 \pm 3.67$ & $-4.68 \pm 2.56$ & $10.73 \pm 4.39$ & 49.3 \\
\hline IS Tau & $-4.55 \pm 2.35$ & $0.45 \pm 1.92$ & $0.21 \pm 1.93$ & $3.24 \pm 2.37$ & $3.91 \pm 3.04$ & 31.7 \\
\hline IW Tau & $-2.50 \pm 0.55$ & $-1.14 \pm 1.91$ & $2.50 \pm 0.47$ & $2.79 \pm 1.80$ & $3.24 \pm 1.97$ & 39.6 \\
\hline $\mathrm{LkH} \alpha 332 / \mathrm{G} 2$ & $2.31 \pm 1.80$ & $7.68 \pm 3.23$ & $-5.58 \pm 2.46$ & $3.72 \pm 2.07$ & $7.36 \pm 3.45$ & 36.5 \\
\hline $\mathrm{LkH} \alpha 332 / \mathrm{G} 1$ & $-1.66 \pm 1.62$ & $0.54 \pm 1.23$ & $2.86 \pm 1.06$ & $5.50 \pm 1.76$ & $3.97 \pm 2.04$ & 32.2 \\
\hline $\mathrm{LkH} \alpha 332$ & $2.91 \pm 3.54$ & $1.21 \pm 1.20$ & $-0.30 \pm 3.69$ & $-0.83 \pm 0.60$ & $2.02 \pm 3.74$ & 47.0 \\
\hline $\mathrm{BD}+26718 \mathrm{~B} \mathrm{Aa}$ & $-6.98 \pm 2.14$ & $5.40 \pm 1.66$ & $-8.26 \pm 2.70$ & $-0.35 \pm 0.12$ & $8.55 \pm 2.71$ & 67.5 \\
\hline $\mathrm{BD}+26718 \mathrm{~B} \mathrm{Bb}$ & $0.98 \pm 3.60$ & $1.06 \pm 1.51$ & $2.07 \pm 3.48$ & $3.02 \pm 1.84$ & $2.55 \pm 3.92$ & 23.3 \\
\hline $\mathrm{BD}+17724 \mathrm{~B}$ & $0.49 \pm 1.64$ & $10.83 \pm 6.20$ & $-0.41 \pm 3.88$ & $-4.02 \pm 5.15$ & $7.44 \pm 6.43$ & 12.8 \\
\hline NTTS 155808-2219 & $0.75 \pm 2.64$ & $-4.06 \pm 2.06$ & $3.43 \pm 2.13$ & $2.31 \pm 1.72$ & $4.13 \pm 3.05$ & 29.4 \\
\hline NTTS 155913-2233 & $1.60 \pm 0.80$ & $-5.38 \pm 2.03$ & $3.09 \pm 0.90$ & $-5.30 \pm 1.85$ & $5.87 \pm 2.11$ & 43.3 \\
\hline NTTS 160735-1857 & $0.89 \pm 2.51$ & $-6.38 \pm 2.24$ & $0.17 \pm 1.91$ & $-6.44 \pm 2.28$ & $6.44 \pm 3.18$ & 43.4 \\
\hline NTTS 160946-1851 & $-1.02 \pm 0.64$ & $0.09 \pm 0.61$ & $0.18 \pm 0.65$ & $1.04 \pm 0.64$ & $1.04 \pm 0.90$ & 30.5 \\
\hline HM Anon & $1.04 \pm 9.33$ & $-7.04 \pm 8.94$ & $-2.65 \pm 7.43$ & $6.61 \pm 6.42$ & $7.12 \pm 11.37$ & 42.1 \\
\hline HN Lup & $7.42 \pm 3.30$ & $-0.94 \pm 3.21$ & $1.57 \pm 3.33$ & $-7.32 \pm 3.15$ & $7.48 \pm 4.60$ & 46.3 \\
\hline RX J1546.1-2804 & $22.34 \pm 1.58$ & $2.86 \pm 4.86$ & $-3.77 \pm 1.84$ & $-24.40 \pm 7.21$ & $23.60 \pm 6.28$ & 14.3 \\
\hline RX J1549.3-2600 & $-0.83 \pm 1.33$ & $-0.45 \pm 1.23$ & $-0.17 \pm 1.40$ & $0.92 \pm 0.46$ & $0.94 \pm 1.64$ & 23.7 \\
\hline RX J1600.5-2027 & $-4.02 \pm 1.61$ & $1.10 \pm 1.42$ & $1.88 \pm 1.69$ & $-3.72 \pm 1.21$ & $4.17 \pm 2.12$ & 28.2 \\
\hline RX J1601.7-2049 & $2.04 \pm 1.75$ & $1.56 \pm 1.73$ & $0.00 \pm 1.37$ & $-2.57 \pm 1.77$ & $2.57 \pm 2.11$ & 29.7 \\
\hline RX J1601.8-2445 & $8.43 \pm 4.55$ & $1.46 \pm 4.76$ & $7.79 \pm 3.53$ & $-3.59 \pm 5.74$ & $8.56 \pm 6.66$ & 13.3 \\
\hline RX J1603.9-2031B & $-2.03 \pm 2.93$ & $-9.58 \pm 1.73$ & $-4.28 \pm 2.11$ & $-8.95 \pm 3.19$ & $9.86 \pm 3.61$ & 15.8 \\
\hline RX J1604.3-2130B & $-2.98 \pm 1.82$ & $-2.52 \pm 1.43$ & $1.03 \pm 1.55$ & $3.78 \pm 1.67$ & $3.91 \pm 2.13$ & 12.3 \\
\hline
\end{tabular}

The velocities of the companions relative to the primaries derived by applying the assumed distances are given in Table 2. They are also plotted in Fig. 2 in cartesian and polar coordinates (similar to Fig. 3 in G95). Our measurements can only cover the projection of motion onto the sky, so the $v_{\rho}$ are given with respect to the main component, not to the observer. The adopted $v$ is the mean of the respective values derived from the fits in cartesian and polar coordinates. In 3 out of 34 systems $v$ is different from zero on the $3 \sigma$ level, in 9 systems on the $2 \sigma$ level and in 18 systems on the $1 \sigma$ level. Thus, we are fairly confident that there really is relative motion of the components in most systems.

\subsection{Origin of the relative motion}

We now examine the origin of this relative motion. For this purpose, we must discriminate orbital motion from an apparent relative motion that can be caused by the proper motion of a $\mathrm{T}$ Tauri star with respect to a background star projected by chance or by the proper motions of two 

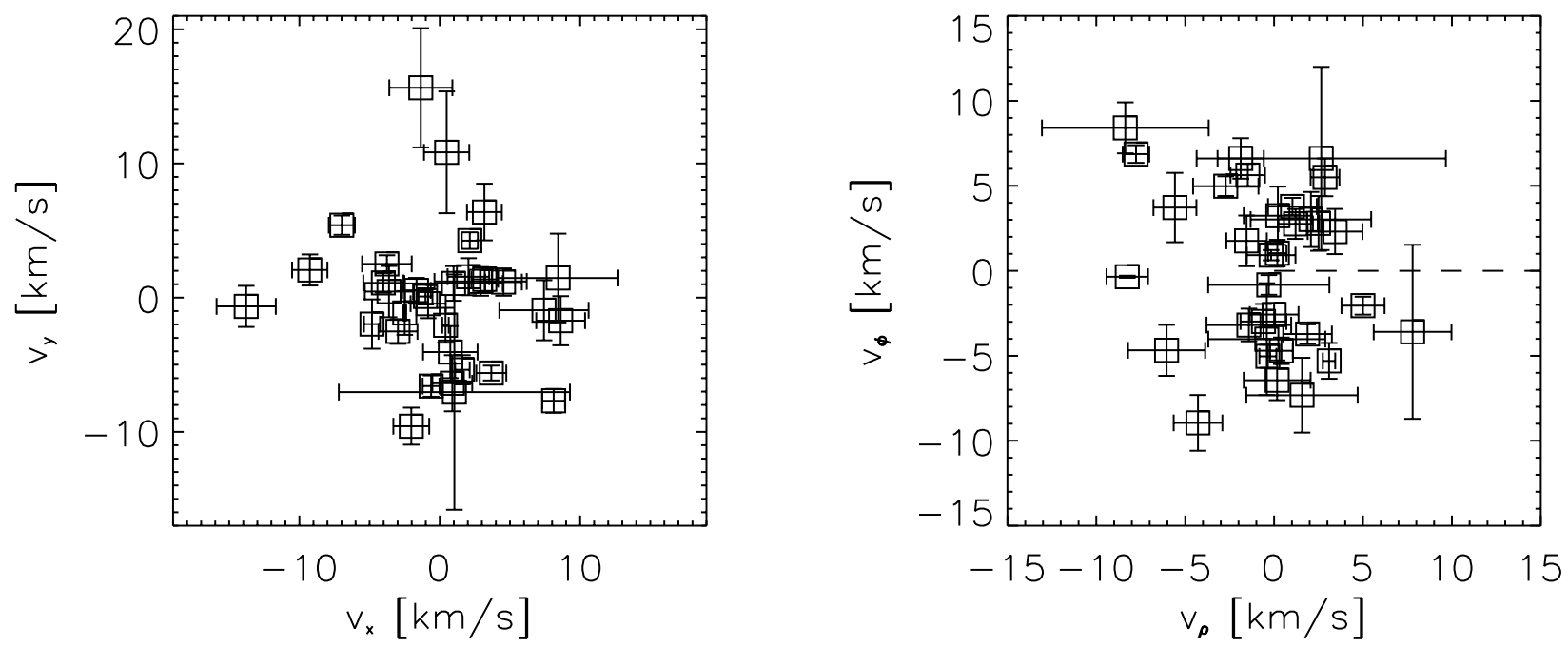

Fig. 2. Relative velocities of the components in T Tauri binary systems in cartesian coordinates (left panel) and polar coordinates (right panel). The dashed line in the right panel points towards the locus of Herbig-Haro objects that is far outside this plot. RX J1546.1-2804 is not plotted in this figure. Its locus is out of the picture in both panels. This plot is similar to Fig. 3 in G95

T Tauri stars projected by chance. One has further to consider that "companions" to T Tauri stars detected with only one observation in one filter are not necessarily stellar and may be Herbig-Haro objects. We will also examine the possible influence of unresolved additional components on the observed motion.

\subsubsection{Orbital motion}

First we will derive an upper limit for relative velocities caused by orbital motion. That limit is given by the condition that in the case of orbital motion the kinetic energy of the system is less than its (negative) potential energy and is equal to it in the extreme case of a parabolic orbit, i.e.

$v^{2} \leq \frac{2 G M_{\text {Sys }}}{r}$,

where $r$ is the instantaneous separation of the components.

We adopt $M_{\text {TTS }} \leq 2 M_{\odot}$ as upper mass limit for one T Tauri star (Hartmann 1998) and take the mean of the measured projected separations as estimate for $r$. There is only one companion with a relative velocity that is larger than the value derived from Eq. (1), namely that of RX J1546.1-2804. The relative velocity of this companion is, however, still consistent with orbital motion, considering the $1 \sigma$ error in its $v$. The lower limit for $v$ is zero because in short pieces of orbit as discussed here the orbital motion may occur purely radial to the observer. Thus, the large majority of the velocities from Table 2 are consistent with orbital motion.

Furthermore, it is interesting to examine the relationship between separation and relative velocity. In the special case of a circular orbit observed face-on this relation will be

$v \propto \frac{1}{\sqrt{a}}$
Table 3. Distribution of the companions with respect to certain relative velocities and separations

\begin{tabular}{lll}
\hline & $d<25 \mathrm{AU}$ & $d \geq 25 \mathrm{AU}$ \\
\hline$v \geq 5 \mathrm{~km} \mathrm{~s}^{-1}$ & $7 \pm 2.6$ & $11 \pm 3.3$ \\
$v<5 \mathrm{~km} \mathrm{~s}^{-1}$ & $3 \pm 1.7$ & $13 \pm 3.6$ \\
\hline
\end{tabular}

for the orbital velocity $v$ and the semimajor axis $a$. Equation (2) will be modified by projection effects and the actual orbital parameters, and the unknown system mass is an additional parameter in this relation. However, close companions should, on average, move faster than distant ones. In Fig. 3 the measured projected velocities, $v$, are plotted as a function of the components' projected separation, $d$. The correlation is weak for the reasons mentioned above, but there is at least a tendency to fulfil the prediction of Eq. (2). Among the closest companions with $d<25 \mathrm{AU}$, relative velocities with $v \geq 5 \mathrm{~km} \mathrm{~s}^{-1}$ are more frequent than for the companions with larger separations (Table 3).

We conclude from this section that the observed relative velocities in almost all cases are not in contradiction to orbital motion. For a final classification, other possible origins of the relative motion must be considered.

\subsubsection{Chance projected background stars}

A background star projected by chance will probably be located at a much larger distance than the observed $\mathrm{T}$ Tauri star, so its proper motion can be neglected. The measured relative motion is thus expected to be the proper motion of the T Tauri star. In Taurus-Auriga there are subgroups with different directions of proper motion and a mean proper motion of $15.8 \mathrm{~km} \mathrm{~s}^{-1}$ (Jones \& Herbig 1979). For Scorpius-Centaurus, de Zeeuw et al. (1999) give 


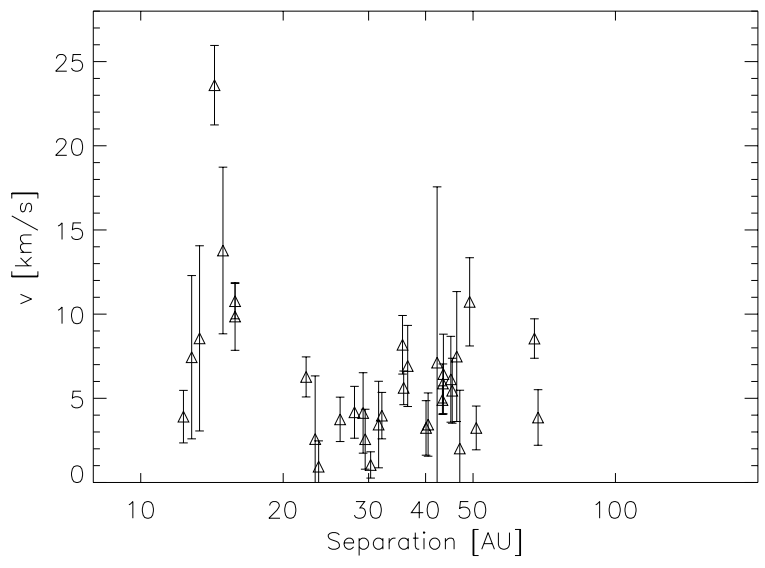

Fig. 3. Relative velocities of the components as function of their mean projected separation

a mean proper motion of $17.6 \mathrm{~km} \mathrm{~s}^{-1}$ with a mean direction of $v_{x}=-17.4 \mathrm{~km} \mathrm{~s}^{-1}$ and $v_{y}=-2.8 \mathrm{~km} \mathrm{~s}^{-1}$. For both SFRs the distances given in Table 1 were adopted. There are only two systems with relative velocities in this order of magnitude, namely V 773 Tau $(v=$ $\left.13.78 \pm 7.34 \mathrm{~km} \mathrm{~s}^{-1}\right)$ and RX J1546.1-2804 $(v=23.60 \pm$ $6.28 \mathrm{~km} \mathrm{~s}^{-1}$ ).

For V $773 \mathrm{Tau}$, the Hipparcos catalogue gives proper motions of $\mu_{x}=-24.89 \pm 1.89 \mathrm{mas} / \mathrm{yr}$ and $\mu_{y}=0.65 \pm$ $2.83 \mathrm{mas} / \mathrm{yr}$. The resultant $v=16.68 \pm 2.28 \mathrm{~km} \mathrm{~s}^{-1}$ is comparable with the observed relative velocity of the components, but the proper motion of V 773 Tau happens almost only in declination (X in Fig. A.1) which is contradictory to our observations. Furthermore, for V 773 Tau the relative motion is still explainable with orbital motion, considering the upper limit derived in Sect. 3.2.1.

In the case of RX J1546.1-2804 there are no Hipparcos data for this individual object, so we adopt the mean proper motion for the OB association Upper Scorpius given by de Zeeuw et al. (1999). These values are $\mu=$ $17.7 \mathrm{~km} \mathrm{~s}^{-1}, \mu_{x}=-17.4 \mathrm{~km} \mathrm{~s}^{-1}$ and $\mu_{y}=-2.8 \mathrm{~km} \mathrm{~s}^{-1}$ with (formal) errors of $\approx 0.1 \mathrm{~km} \mathrm{~s}^{-1}$. The respective values from Table 2 are $v=23.6 \pm 6.3 \mathrm{~km} \mathrm{~s}^{-1}, v_{x}=22.3 \pm$ $1.6 \mathrm{~km} \mathrm{~s}^{-1}$ and $v_{y}=2.9 \pm 4.9 \mathrm{~km} \mathrm{~s}^{-1}$. This is a close correspondance, given that the the proper motion presented by de Zeeuw et al. (1999) is not for this single object, but for the whole association. Note that $\boldsymbol{\mu}$ and $\boldsymbol{v}$ must be antiparallel if the companion is a chance-projected background star, because in that case $\boldsymbol{\mu}$ is the motion of the primary with respect to the "companion". Another argument that supports the idea that the companion of RX J1546.1-2804 is a chance-projected background star is that its relative motion is above the limit given by Eq. (1). It is, however, still consistent with orbital motion at the $1 \sigma$ level.

One must take into account that in both cases the measured projected separations are $d \approx 00^{\prime \prime} 1$, which makes any chance projections very unlikely (see Sect. 3.2.3 for the probability of chance projections). Thus, one must consider other origins of these high relative velocities. One solution may be that the distances of individual systems are largely different from the adopted values (Table 1) for the SFRs as a whole. The unusually high derived values for relative velocities would then be caused by overestimating the objects' distances. Another possibile explanation, namely the presence of unresolved additional companions, will be discussed in Sect. 3.2.5.

The companion of RXJ1546.1-2804 may be such a chance-projected background star. In the case of V 773 Tau this seems to be unlikely because the direction of proper motion does not match, however, the high relative velocity of the components remains problematic. Further observations of these systems will be necessary to determine whether there is a curvature in the relative motion which would undoubtedly classify it as orbital motion. In general, chance-projected background stars are not frequent among close visual companions to T Tauri stars.

\subsubsection{Chance projection of two $T$ Tauri stars}

There may also be companions that are in fact objects projected by chance belonging to the same SFR as the "primary". In that case both components would have roughly the same proper motion. Any relative motion would then be caused by the velocity dispersion within the respective SFR or subgroup, as was mentioned by G95. These velocity dispersions are between 1 and $2 \mathrm{~km} \mathrm{~s}^{-1}$ in each coordinate for different subgroups in Taurus-Auriga (Jones \& Herbig 1979). For Scorpius-Centaurus, de Zeeuw et al. (1999) formally derived a velocity dispersion of only $0.1 \mathrm{~km} \mathrm{~s}^{-1}$. One cannot distinguish this kind of relative motion from slow orbital motion by analyzing relative velocities.

It is, however, not probable that chance projections within the same SFR are a frequent phenomenon, because of the low stellar density in the SFRs discussed here. Leinert et al. (1993) concluded that there are less than $410^{-5}$ objects brighter than $K=12$ mag per $\operatorname{arcsec}^{2}$ in Taurus-Auriga. This includes association members and field stars. All companions discussed here are brighter than $K=12 \mathrm{mag}$ and have projected separations of less than $0 \prime \prime 5$. The mean number of chance-projected companions within a radius of 0.5 around our 21 objects in Taurus-Auriga is thus

$N_{\text {proj }}=21 \cdot 0.5^{2} \cdot \pi \cdot 410^{-5}=6.610^{-4}$.

For Scorpius-Centaurus, Köhler et al. (2000) derived a stellar density of $(6.64 \pm 0.45) \quad 10^{-4} \mathrm{arcsec}^{-2}$ from star counts in the vicinity of X-ray selected $\mathrm{T}$ Tauri stars. Applying Eq. (3) yields a mean number of $5.710^{-3}$ chanceprojected companions around 11 objects in ScorpiusCentaurus. These values are low enough to rule out that chance projections within the same SFR cause the detection of an appreciable number of close "companions". The low observed relative velocities in some systems are probably due to the fact that we can only detect the projected motion on the sky. If most of the orbital motion happens radial to the observer in the time span covered by our data it will not be measurable by our observations. 


\subsubsection{Herbig-Haro objects}

G95 raised the question of whether an appreciable number of companions of young stars detected with only one observation in one broad band filter may in fact not be stellar, but rather condensations in gaseous nebulae. Such companions would appear as Herbig-Haro objects that are driven by strong winds of active T Tauri stars. They usually have velocities of some $100 \mathrm{~km} \mathrm{~s}^{-1}$ (e.g. Schwartz 1983). Moreover, their relative motion with respect to their driving source can be only radial, not tangential. Thus, the locus of Herbig-Haro objects in Fig. 2 (right panel) is in the direction of the dashed line in this figure, but far outside the plot. As was reported by G95, we find no system where the observed relative velocity is consistent with these restrictions.

Furthermore, in the case of HV Tau, where companion $\mathrm{C}$ has been declared as a Herbig-Haro object, we could show that in fact it is a stellar companion (Woitas \& Leinert 1998).

\subsubsection{Apparent relative motion caused by unresolved companions}

Another problem with observed relative velocities in multiple systems has been pointed out by G95. If the main component has an additional unresolved companion, orbital motion in this close pair can shift the photocenter of the "primary". This will be misinterpreted as motion of the visual secondary if only relative astrometry is measured, as is the case here. G95 have used this effect to explain the surprisingly high relative velocity in the Elias 12 system where the visual companion is moving with $10.73 \mathrm{~km} \mathrm{~s}^{-1}$ at a separation of $49.3 \mathrm{AU}$ (Table 2). This is only slightly below the upper limit for orbital motion, which is $12.0 \mathrm{~km} \mathrm{~s}^{-1}$ in this case (Eq. (1)). In this system, the primary has another companion with a separation of $d=23$ mas, detected by Simon et al. (1995) using lunar occultations. If the system mass of the close pair is assumed to be $1 M_{\odot}$ and a relation $\langle d\rangle=0.95 a$ between the mean projected separation $\langle d\rangle$ and the semi-major axis a (Leinert et al. 1993) is adopted, one would expect the period of the close pair to be $P \approx 6.4 \mathrm{yr}$. This is roughly the same timespan that is covered by our observations (Table A.1). The shift of the photocenter of the close pair with respect to the visual secondary will cancel, and thus the derived secondary's velocity is not affected by the tertiary.

Also in the V 773 Tau system, where we have noticed an unusually high relative velocity of the visual secondary (Sect. 3.2.2) there is an additional spectroscopic companion (Welty 1995). The period of this close pair is 51.1 days, so possible shifts of the photocenter are less than 2 mas and thus not measurable by our observations.

A candidate for a system where the observed relative motion may be influenced by an unresolved tertiary is $\mathrm{BD}+26718 \mathrm{~B} \mathrm{Aa}$. In this system $v=8.55 \mathrm{~km} \mathrm{~s}^{-1}$ at a separation of $67.5 \mathrm{AU}$. Similar to Elias 12, this value is close to the upper limit for orbital motion $\left(v \leq 10.2 \mathrm{~km} \mathrm{~s}^{-1}\right.$ from Eq. (1)), but also far below the relative velocity expected for a background star projected by chance (Sect. 3.2.2).

\subsubsection{Conclusions}

The observed relative velocties can be explained by orbital motion. Together with the result that other origins of relative motion can be ruled out at a high confidence level for most systems, we conclude that the observed motion is orbital motion in nearly all binaries discussed.

\subsection{Estimation of an empirical average system mass}

For all binary systems discussed here the available portions of the orbit are too short to calculate orbital parameters. The results presented in Table A.1, however, remain valuable for future orbit determinations that will yield empirical masses for $\mathrm{T}$ Tauri binary systems. Furthermore, it is already possible to estimate an average system mass from this database. This average mass is not dependent on theoretical assumptions about the physics of PMS evolution and should therefore be a reliable empirical estimation of the masses of $\mathrm{T}$ Tauri stars. To derive this mass we follow the approach of G95, but improve it in some important aspects.

First, we write Kepler's third law in the natural units $M / M_{\odot}, a / 1 \mathrm{AU}$ and $P / 1 \mathrm{yr}$ :

$M_{\text {Sys }}=\frac{a^{3}}{P^{2}}$

and assume a face-on circular orbit where the total orbital motion happens tangential to the observer and therefore equals the observed velocity:

$v_{\text {face-on, } \operatorname{circ}}=\frac{2 \pi a}{P}$.

In this special case $\left(i=0^{\circ}, e=0\right)$ Eq. (4) becomes

$M_{\text {Sys }}=\frac{v_{\text {face-on,circ }}^{2} a}{4 \pi^{2}}$.

Unfortunately, we don't know $a$ or $v_{\text {face-on,circ }}$ of our systems. G95 used computer simulations of binary orbits to derive statistical relations between the observed quantities $d_{\text {proj }}$ and $v_{\text {obs }}$ on the one hand and $a$ and $v_{\text {face-on,circ }}$ on the other hand. They assumed an eccentricity distribution of $f(e)=2 e$ and an isotropic distribution of inclinations and obtained the following results:

$$
\begin{aligned}
& <d_{\text {proj }}>=0.91 a . \\
& <v_{\text {obs }}>=0.72 v_{\text {face-on,circ }} .
\end{aligned}
$$

However, $d_{\text {proj }}$ and $v_{\text {obs }}$ are not uncorrelated. For example, if we observe a companion in the outer part of its eccentric orbit, $d_{\text {proj }}$ will be larger, and $v_{\text {obs }}$ smaller than their average values. Therefore, we cannot insert these equations 
into Eq. (6) to obtain a statistical relation between $d_{\text {proj }}$, $v_{\text {obs }}$, and $M_{\text {Sys }}$.

To overcome this problem, we performed more sophisticated computer simulations. Each simulation contains 10 million binaries with a fixed system mass and randomly distributed orbital parameters. The periods follow the distribution of periods of main-sequence stars (Duquennoy \& Mayor 1991), the distribution of eccentricities is $f(e)=2 e$ and the inclinations are distributed isotropically, while all the other parameters have uniform distributions. The distances to the observer are varied within a range of $143 \pm 25$ pc. We chose two observation dates separated by a random timespan between 4 and 10 years and computed the average projected separation and orbital velocity, in much the same way as we did for the real data. We then select binaries in the projected separation range from 10 to $70 \mathrm{AU}$. For these binaries, we compute $<v^{2} \cdot d>/ M_{\text {Sys }}$. These simulations are repeated for different system masses. For $M_{\text {Sys }}$ between $0.5 M_{\odot}$ and $2.5 M_{\odot}$, the results vary from 18.4 to $18 \mathrm{AU}^{3} /\left(\mathrm{yr}^{2} M_{\odot}\right)$. This gives us the following relation:

$M_{\mathrm{Sys}} \approx \frac{1}{18.2} \cdot\left\langle v^{2} \cdot d\right\rangle$

The term on the right hand side of Eq. (9) contains $v^{2}$. Since the measured velocities and separations differ from the real values by some unknown measurement error, this leads to an additional bias term:

$$
\begin{aligned}
<v_{\mathrm{obs}}^{2} \cdot d_{\mathrm{obs}}> & =\left\langle\left(v_{\text {real }}+\delta v\right)^{2} \cdot\left(d_{\text {real }}+\delta d\right)>\right. \\
& =\left\langle v_{\text {real }}^{2} \cdot d_{\text {real }}>+<\delta v^{2} \cdot d_{\text {real }}>\right.
\end{aligned}
$$

Combining Eqs. (9) and (10), and using $\Delta v$ as an estimate for $\delta v$, we obtain a relation that allows us to use the observed values to obtain an average system mass:

$$
M_{\mathrm{Sys}} \approx \frac{1}{18.2}\left(<v_{\mathrm{obs}}^{2} \cdot d_{\mathrm{proj}}>-<\Delta v_{\mathrm{obs}}^{2} \cdot d_{\mathrm{proj}}>\right) .
$$

This is only an estimate for the mass in an ensemble of systems with identical system masses. The stars in our sample will probably not all have the same mass. However, we expect them to have similar masses, and for a sufficiently large number of systems, the uncertainties will cancel. The result should yield a reliable average mass for these systems.

If we use the data of all stars in our sample, Eq. (11) yields a system mass of $2.5 M_{\odot}$. Excluding RX J1546.12804 lowers the result to $2.0 M_{\odot}$. If we further exclude V 773 Tau, Elias 12, and BD+26718B Aa, we arrive at a system mass of $1.3 M_{\odot}$. We have reason to assume that the companion to RX J1546.1-2804 is a chance projected background star. For the three other systems mentioned, the velocity is puzzling at first sight, but is still consistent with orbital motion. Furthermore, other possible explanations fail for V 773 Tau and Elias 12 (Sects. 3.2.2 and 3.2.5). Thus, it does not seem justified to exclude them from the sample, and we will adopt $2 M_{\odot}$ as the result for the average dynamical system mass.

Given the statistical uncertainties, it is difficult to estimate the error of the system mass. Using the standard deviation of the quantities averaged in Eq. (11) to estimate the error of the mean yields $\approx 0.7 M_{\odot}$. This is in agreement with the scatter we obtain if we exclude or include the stars mentioned in the last paragraph.

We conclude that the average mass of the systems in our sample is in the range $1.3 \ldots 2.5 M_{\odot}$, with a most probable system mass of $2 M_{\odot}$. Our result is thus consistent with the expectation that $\mathrm{T}$ Tauri stars' masses are around $M \approx 1 M_{\odot}$ and also with the average mass of 1.7 $M_{\odot}$ that G95 derived for their sample.

\section{Discussion}

\subsection{Comparision with theoretical results}

We have estimated masses for the components by comparison with theoretical PMS models for a subsample that contains 17 out of our 34 systems. In these cases, we obtained resolved $J$ band photometry. This spectral band is supposed to be least affected by circumstellar excess emission and can thus be taken as an indicator of stellar luminosity. Moreover, in these 17 systems, there are no additional companions known, so the mass sum of the components derived from theoretical models will match the dynamical system masses. We placed the components of these systems into the HRD, estimating the stellar luminosity from the resolved $J$ band magnitudes, assigning the optical spectral type of the system (taken from Kenyon \& Hartmann 1995 for Taurus-Auriga and Walter et al. 1994 for Scorpius-Centaurus) to the primary and assuming that all components within one system are coeval. Masses of the components were then derived using the PMS evolutionary tracks of D'Antona \& Mazzitelli (1998), Swenson et al. (1994) and Baraffe et al. (1998).

The mean mass obtained for this subsample from the HRD is $0.88 M_{\odot}$ for the D'Antona \& Mazzitelli (1998) tracks and $1.17 M_{\odot}$ for the Swenson et al. (1994) tracks. The PMS model from Baraffe et al. (1998) yields a mean mass of $1.28 M_{\odot}$. The uncertainties of the mass estimates that originate from observational data used for placing the components into the HRD are $\approx 0.1 M_{\odot}$. The average empirical mass derived from Eq. (11) for that subsample ${ }^{1}$ is $1.22 \pm 0.50 M_{\odot}$. Within the large formal error, the predictions of all three PMS models match our empirical result. However, our dynamical $\langle M\rangle$ is much closer to the mean masses derived using the Baraffe et al. (1998) and Swenson et al. (1994) tracks than to the value calculated from the D'Antona \& Mazzitelli (1998) PMS model, which

\footnotetext{
${ }^{1}$ For the reasons mentioned above, the subsample does not contain RXJ1546.1-2804, V 773 Tau, Elias 12 and $\mathrm{BD}+26718 \mathrm{~B}$ Aa. Thus, the mean mass for the subsample is lower than the value derived in Sect. 3.3.
} 
seems to underestimate T Tauri star masses. This finding has been recognized by other authors. Bonnell et al. (1998) estimated T Tauri stars' masses from infall velocities of accreted material and also conclude that the empirical masses are generally larger than those predicted by the D'Antona \& Mazzitelli (1998) model. A similar result was reported by Simon et al. (2000), who calculated T Tauri stars' masses from Keplerian motion in circumstellar and circumbinary disks.

\subsection{Implications for binary statistics}

There is significant relative motion in most systems, and this motion is in almost all cases consistent with orbital motion (Sect. 3). As already pointed out by G95, this demonstrates that the large majority of all close companions detected in the multiplicity surveys mentioned in Sect. 2.1 really are gravitationally-bound stars. No binary component discussed here has to be reclassified as a Herbig-Haro object (Sect. 3.2.4), and only 2 out of 34 companions may be chance projected background stars (Sect. 3.2.2).

This finding is particularly important because there is a companion overabundance among $\mathrm{T}$ Tauri stars in the SFRs discussed here when compared to main-sequence stars in the solar neighbourhood (Leinert et al. 1993; Ghez et al. 1993; Ghez et al. 1997a; Köhler \& Leinert 1998; Köhler et al. 2000). In Taurus-Auriga, almost all T Tauri stars seem to be components of multiple systems. To further confirm this result, Köhler \& Leinert (1998) performed stellar counts in the vicinity of their survey objects and concluded that in Taurus-Auriga, statistically 4.3 out of 44 apparent companions are projected background objects. Köhler et al. (2000), in a similar way, derived a number of 7.8 chance projections per 46 companions in Scorpius-Centaurus. We found one candidate for a chance-projected background star out of 21 objects in Taurus-Auriga and one candidate out of 11 objects in Scorpius-Centaurus. The results are not directly comparable because we have only studied the closest pairs for which chance projections are least probable. The percentage of background stars projected by chance among the observed companions is, however, of the same order of magnitude in both studies. Thus, it can be concluded that chance projections do not affect the binary statistics significantly in the SFRs discussed here.

Köhler et al. (2000) excluded six close companions in Scorpius-Centaurus from a restricted sample. Their observed separations are less than the strict diffraction limit $\lambda / D=0$ ". 13 of a $3.5 \mathrm{~m}$-telescope in the $K$-band, so they cannot be definitely distinguished from elongated single objects. In three of these cases, namely RX J1601.8-2445, RX J1603.9-2031B and RX J1604.3-2130B we derive a relative velocity that is consistent with orbital motion (see Sect. 3). Thus, we propose to classify these objects as binary systems in further studies of multiplicity in the OB association, Scorpius-Centaurus.

\section{Summary}

Based on repeated measurements of the relative astrometry in 34 close $\mathrm{T}$ Tauri binary systems, we have reproduced the results given by G95 and extended their work to a larger number of binaries and particularly to a much longer timespan of up to 10 years. We showed that in most systems significant relative motion of the components has occured. In almost all cases this relative motion can be explained by orbital motion. In only two systems the observed motion may be the result of the proper motion of a $\mathrm{T}$ Tauri star that is accidentally projected in the close vicinity of a background star.

From the short pieces of orbit available at the moment (up to $20^{\circ}$ in position angle), we derive a mean dynamical system mass of $2.0 \pm 0.7 M_{\odot}$ for our sample. This mass is consistent with the predictions of current sets of PMS evolutionary models within the uncertainties. The large formal error of this mean mass does not allow a significant discrimination between different models, but we draw the tentative conclusion that the masses predicted by the D'Antona \& Mazzitelli (1998) model may be systematically too low.

The result that orbital motion can be detected in most systems discussed here indicates that the "companions" found in previous multiplicity surveys really are gravitationally bound stars. This is a further confirmation of the binary overabundance in Taurus-Auriga and Scorpius-Centaurus compared to nearby main sequence stars. Furthermore, the detection of orbital motion allows a definite classification of three objects with very close separations as stellar companions.

Acknowledgements. We thank the staff at ESO La Silla and Calar Alto for their support during several observing runs. In particular we are grateful to Andreas Eckart and Klaus Bickert for their support in observing with the SHARP I camera. We modified a program written by Sabine Frink to carry out the computer simulations described in Sect. 3.3. The authors appreciate fruitful discussions with Michal Simon, and thank the anonymous referee for fair and constructive criticism.

\section{Appendix A: Overview of observations}

Table A.1 presents an overview of all measurements of the relative astrometry in $\mathrm{T}$ Tauri binary systems that form the database for the present study. In Fig. A.1 these measurements are plotted as a function of epoch for the individual systems in cartesian and polar coordinates. 

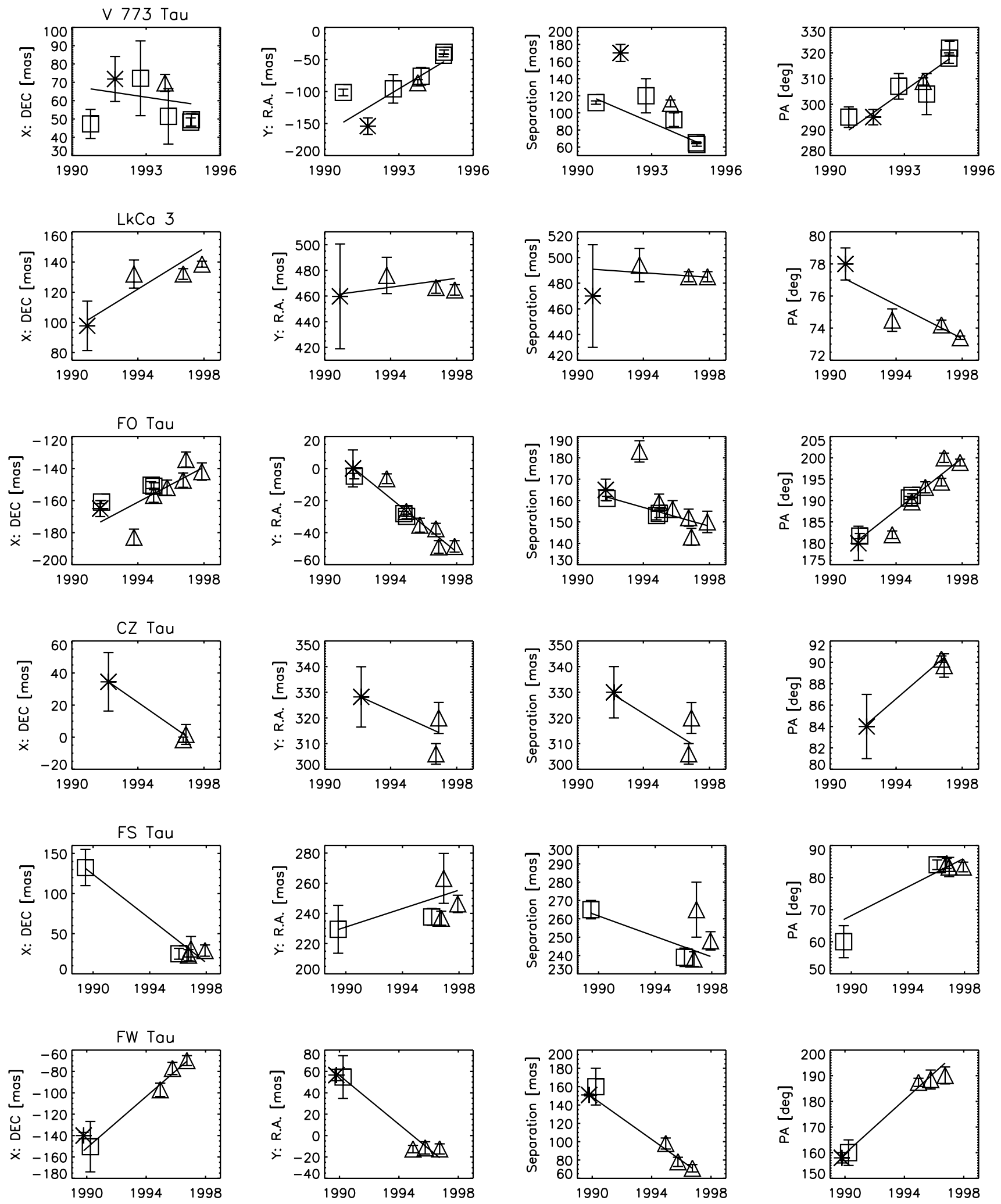

Fig. A.1. Relative astrometry of the components in T Tauri binary systems in cartesian coordinates (first and second column) and polar coordinates (third and fourth column). The solid lines indicate the results of weighted linear fits to this data. Triangles denote our new data presented in this paper. Asterisks refer to measurements of "first epoch" that coincide with the detection of the companions. They have already been published (Leinert et al. 1993; Köhler \& Leinert 1998). Squares indicate data points taken from literature, in most cases from G95 (see Table A.1 for reference) 

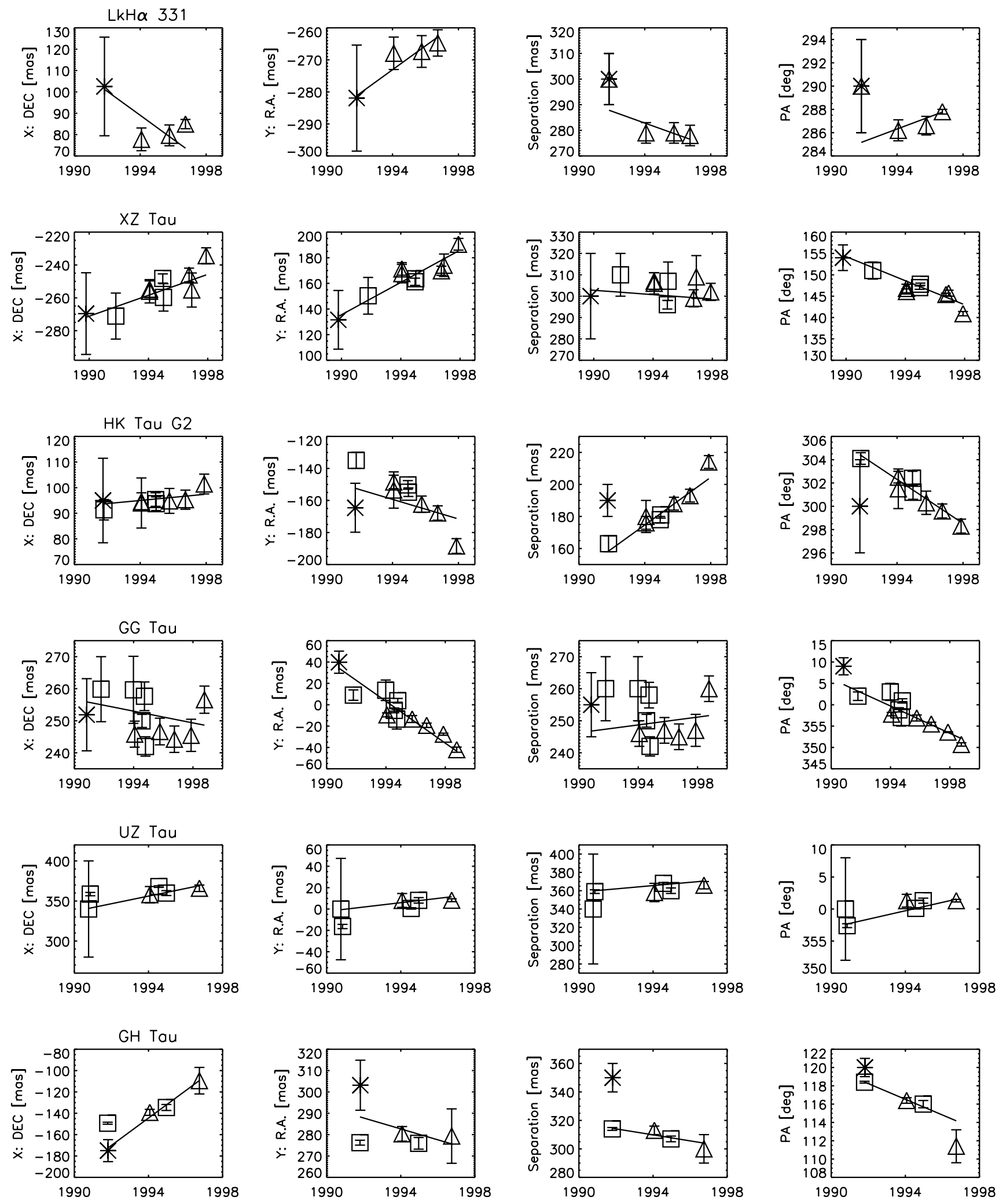

Fig. A.1. continued 

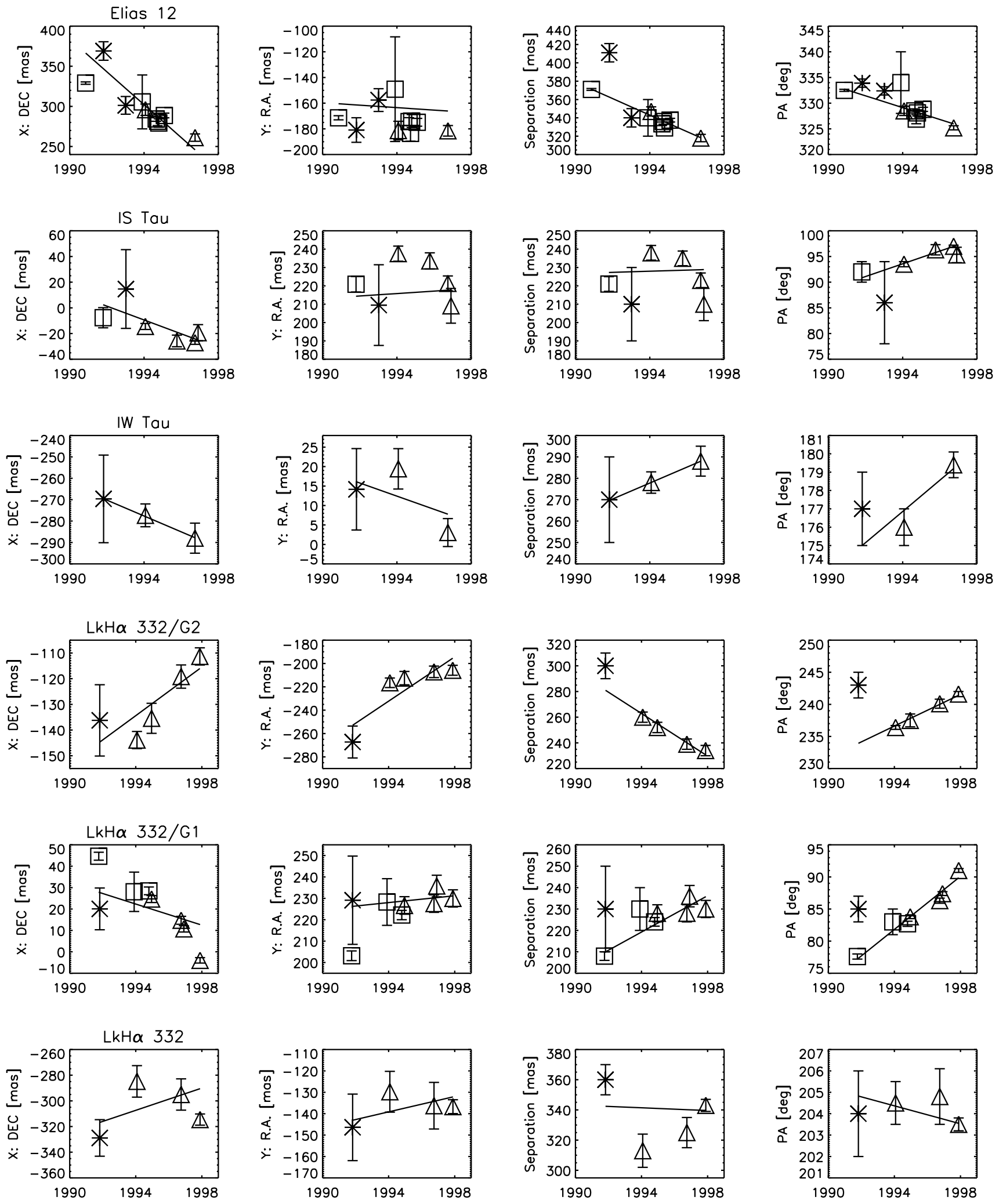

Fig. A.1. continued 

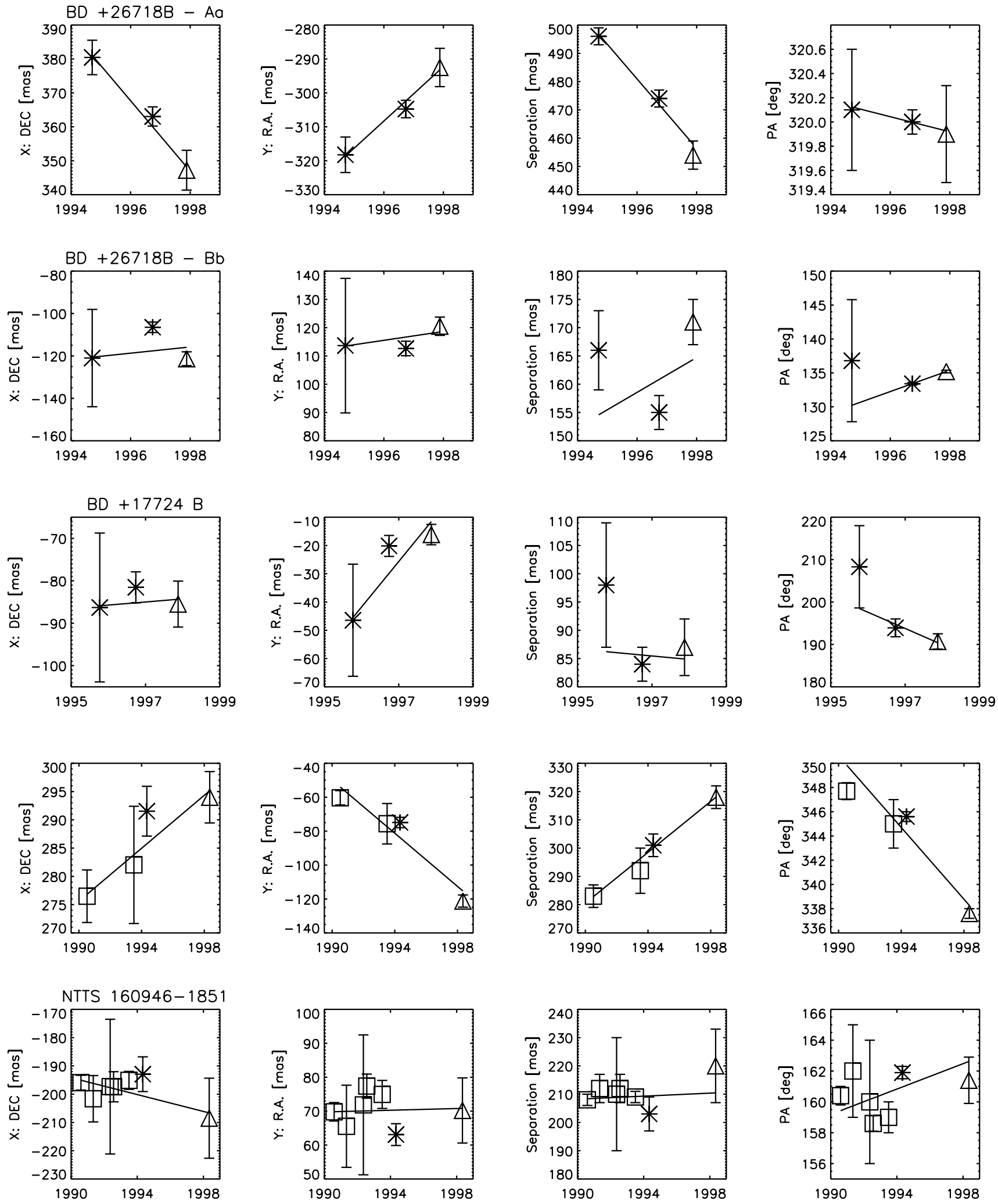

Fig. A.1. continued 


\section{References}

Baraffe, I., Chabrier, G., Allard, F., \& Hauschildt, P. H. 1998, A\&A, 337, 403

Bonnell, I. A., Smith, K. W., Meyer, M. R., et al. 1998, MNRAS, 299, 1013

Casey, B. W., Mathieu, R. D., Vaz, L. P. R., Andersen, J., \& Suntzeff, N. B. 1998, AJ, 115, 1617

Covino, E., Catalano, S., Frasca, A., et al. 2000, A\&A, 361, L49

D'Antona, F., \& Mazzitelli, I. 1994, A\&AS, 90, 467

D'Antona, F., \& Mazzitelli, I. 1998, PASPC, 134, 442

Duquennoy, A., \& Mayor, M. 1991, A\&A, 248, 455

Ghez, A. M., Neugebauer, G., \& Matthews, K., 1993, AJ, 106, 2005

Ghez, A. M., Weinberger, A. J., Neugebauer, G., Matthews, K., \& McCarthy, D. W. 1995, AJ, 110, 753 (G95)

Ghez, A. M., McCarthy, D. W., Patience, J., \& Beck, T. 1997a, ApJ, 477, 705

Ghez, A. M., White, R. J., \& Simon, M. 1997b, ApJ, 490, 353

Hartmann, L. 1998, Accretion Processes in Star Formation (Cambrigde University Press, Cambridge)

Hofmann, R., Blietz, M., Duhoux, Ph., et al. 1992, SHARP and FAST: NIR Speckle and Spectroscopy at the MPE, in ed. M. H. Ulrich, ESO Conference and Workshop Proceedings, vol. 42,617

Jones, B. F., \& Herbig, G. H. 1979, AJ, 84, 1872

Kenyon, S. J., \& Hartmann, L. 1995, ApJS, 101, 117

Knox, K. T., \& Thompson, B. J. 1974, ApJ, 193, L45

Köhler, R., \& Leinert, Ch. 1998, A\&A, 331, 977

Köhler, R., Kunkel, M., Leinert, Ch., \& Zinnecker, H. 2000, A\&A, 356, 541

Krist, J. E., Burrows, J., Stapelfeldt, K. R., et al. 1997, ApJ, 481,447
Krist, J. E., Stapelfeldt, K. R., Burrows, C. J., et al. 1998, ApJ, 501, 841

Leinert, Ch., \& Haas, M. 1989, A\&A, 221, 110

Leinert, Ch., Zinnecker, H., Weitzel, N., et al. 1993, A\&A, 278, 129

Lohmann, A. W., Weigelt, G., \& Wirnitzer, B. 1983, Appl. Opt., 22, 4028

Mathieu, R. D., Ghez, A. M., Jensen, E., et al. 2000, in Protostars and Planets IV, ed. V. Mannings, A. P. Boss, \& S. S. Russell (The University of Arizona Press, Tucson, Arizona)

McCaughrean, M. J., \& Stauffer, J. R. 1994, AJ, 108, 1382

Palla, F., \& Stahler, S. W. 1999, ApJ, 525, 772

Press, W.H., Teukolsky, S. A., Vetterling, W. T., et al. 1994, Numerical Recipes in C (Cambridge University Press, Cambridge)

Roddier, C., Roddier, F., Northcott, M. J., et al. 1996, ApJ, 463,326

Schwartz, R. D. 1983, ARA\&A, 21, 209

Simon, M., Chen, W. P., Howell, R. R., Benson, J. A., \& Slowik, D. 1992, ApJ, 384, 212

Simon, M., Ghez, A. M., Leinert, Ch., et al. 1995, ApJ, 443, 625

Simon, M., Holfeltz, S. T., \& Taff, L. G. 1996, ApJ, 469, 890

Simon, M., Dutrey, A., \& Guilloteau, S. 2000, ApJ, 545, 1034

Swenson, F. J., Faulkner, J., Rogers, F. J., \& Iglesias, C. A. 1994, ApJ, 425, 286

Walter, F. M., Vrba, F. J., Mathieu, R. D., Brown, A., \& Myers, P. C. 1994, AJ, 107, 692

Welty, A. D. 1995, AJ, 110, 776

Wichmann, R., Bastian, U., Krautter, J., Jankovics, I., \& Rucińsky, S. M. 1998, MNRAS, 301, L39

Woitas, J., \& Leinert, Ch. 1998, A\&A, 338, 122

de Zeeuw, P. T., Hoogerwerf, R., de Bruijne, J. H. J., Brown, A. G. A., \& Blaauw, A. 1999, AJ, 117, 354 